\title{
Acrónimos y compuestos en publicidad escrita: una tendencia del español de Chile actual
}

\author{
Marcela Cabrera Pommiez* \\ Universidad de Chile
}

\begin{abstract}
Resumen
Los textos publicitarios y propagandísticos han ido paulatinamente incorporando dos tipos de construcciones que resultan bastante funcionales en términos de economía lingüística. Se trata de 'compuestos léxicos' y de 'acrónimos', los que surgen debido a la necesidad de presentar ciertos segmentos de información de manera breve y concentrada. Estas palabras mantienen en la superficie textual solo lexemas nucleares y eliminan conectores intrafrásticos, características propias de una redacción más extensa. El presente estudio se centró en el examen de avisos publicitarios, letreros, boletas de servicios y otros textos dirigidos a un receptor masivo. Luego de la recolección y análisis de nuevos compuestos y acrónimos, éstos son descritos en términos morfológicos, léxicos y gramaticales. El estudio pone de manifiesto una revitalización de la composición y la acronimia en el ámbito de textos escritos breves y de difusión masiva.
\end{abstract}

* Para correspondencia, dirigirse a: Marcela Cabrera Pommiez (mecabrera@uchile.cl), DEMRE (Departamento de Evaluación, Medición y Registro Educacional), Universidad de Chile, Av. José Pedro Alessandri 685, Ñunoa, Santiago, Chile. 
Palabras clave: lexicogenesia, acronimia, composición, lenguaje publicitario.

\author{
ACRONYMS AND COMPOUNDS IN WRITTEN ADVERTISING: A TREND IN \\ CURRENT Chilean Spanish
}

\begin{abstract}
Written texts in advertising have gradually introduced two types of construction which are quite functional in terms of linguistic economy: compound words and acronyms. They are created in order to present certain units of information in a brief and condensed manner. These constructions perform this task by maintaining on the textual surface only nuclear lexemes and by deleting the normal connective elements. The study focused on advertisements, placards, sales checks, and other texts intended for public addresses. It involved collecting and analyzing new compound words and acronyms morphologically, lexically and syntactically. The study makes manifest a revitalization of the use of both lexical compounds and acronyms in brief written texts of wide circulation.
\end{abstract}

Key words: lexicogenesis, acronyms, compounding, the language of advertising.

Recibido: 31/08/10. Aceptado: 09/05/11.

\title{
1. INTRODUCCIÓN
}

A partir de la observación de carteles, boletas de servicios públicos y de variados avisos publicitarios se puede identificar un fenómeno lingüístico que se ha ido extendiendo en el discurso tanto publicitario como propagandístico y que constituye una novedad en la descripción del español escrito de Chile actual. Se trata de compuestos y acrónimos surgidos recientemente, creados a partir de grupos de palabras que podrían aparecer en una frase u oración, con lo cual exigirían, por tanto, una redacción más extensa y un espacio mayor para su escritura. Es el caso de fonoservicio (escrito con variantes, como Fono servicio o FONOSERVICIO), fonoayuda, ChileInventa o fotoborde. Se trata de una tendencia a la economía y a la rapidez comunicativas; características 
propias de textos escritos breves y dirigidos hacia un público masivo, por lo cual es pertinente sintetizar el mensaje, a través de un compuesto o de un acrónimo, debido a que ambos mantienen en la superficie textual sus constituyentes nucleares, con la ventaja de que eliminan preposiciones y otros nexos que se usarían en una redacción más extensa (como teléfono que ofrece servicios, en el caso de fonoservicio). Es responsabilidad del receptor establecer el vínculo lógico que une a los miembros del compuesto o acrónimo y determinar, así, el verdadero sentido pragmático de la expresión sintetizadora. El objetivo del presente estudio es dar cuenta de una tendencia del español de Chile actual, consistente en la productividad tanto de la composición como de la acronimia en textos escritos de difusión masiva. Se trata de recursos poco estudiados en la lingüística hispánica actual, debido a que existe el prejuicio de que son recursos poco relevantes en comparación con otros procedimientos como la derivación y, especialmente, la sufijación. Cabe destacar que nuestro trabajo es descriptivo, es decir, busca delimitar un par de recursos lexicogenésicos y exponer sus características gramaticales, léxicas, sintácticas y discursivas.

\section{MARCO TEÓRICO}

\section{El LENGUAJE DE LA PUBLICIDAD}

En general, se puede definir la publicidad como el conjunto de técnicas utilizadas para divulgar un anuncio comercial a través de los medios de comunicación social como la prensa y la televisión ${ }^{1}$. El fin último de estos anuncios es promover el consumo de un producto o servicio por parte de los sujetos a los que se dirigen. Es por esta razón que la publicidad, antes que un fin denotativo o informativo, posee un fin altamente connotativo o persuasivo, pues, en mayor o menor medida, toda publicidad busca resaltar las virtudes del producto o servicio anunciado, para así estimular su consumo. No obstante, la publicidad no resalta solamente las cualidades útiles, materiales o estéticas de lo anunciado, sino que frecuentemente apela a una cierta dimensión axiológica asociada a los bienes:

\footnotetext{
1 En rigor, esta es la publicidad comercial, que se distingue de la publicidad institucional, la que se encuentra, por ejemplo, en anuncios de prevención de SIDA (Ferraz 2000: 9).
} 
La publicidad se apoya, más aún que en los valores de uso objetivos, en valores de carácter simbólico asociados de forma estable a los productos: estos se convierten en signos sociales de distinción, éxito, juventud, modernidad, atracción erótica, etc. En este sentido, puede decirse que la publicidad no sólo difunde sino que también conforma los valores sociales de la ideología y 'mitología' de nuestro tiempo (Ferraz 2000: 10).

La publicidad se distingue de la propaganda, cuyo objetivo principal no es vender un producto, sino difundir información relevante para el bien común. Debido a que sus estrategias son las mismas que las de la publicidad, también se incluye a la propaganda en este estudio. Ferraz (2000) señala que las dos principales vías para la obtención de la persuasión mediante la lengua son la implicación de los receptores y la exaltación de los productos. La primera vía funciona mediante las oraciones imperativas, las modalidades, las formas de tratamiento, entre otros medios; mientras que la segunda vía lo hace a través de recursos intensificadores como la gradación del adjetivo.

Por otra parte, entre las características discursivas de la publicidad se encuentran la rapidez, la brevedad y la eficacia de los anuncios ${ }^{2}$. Además, la publicidad recoge los más variados registros y medios de expresión, así como también incorpora toda clase de innovaciones lingüísticas, para, de este modo, captar la atención de los más heterogéneos receptores. De lo anterior se desprende que la publicidad utilice, ente otras estrategias, los neologismos, los extranjerismos, los recursos retóricos como la rima, la hipérbole o la paronomasia, las condensaciones, las elisiones y los compuestos. Este último recurso es efectivo en cuanto a su economía informativa, pues permite entregar, de manera veloz y condensada, gran cantidad de información y, a la vez, con grandes cargas connotativas, pues la elisión de elementos como las preposiciones amplía el espectro de relaciones posibles entre los morfemas que forman el compuesto. Además, muchas veces los compuestos llevan incorporados en alguno de sus morfemas otros procesos como la abreviación o la incorporación de un extranjerismo, como en eco-pack (en lugar de paquete ecológico), lo que plantea delimitar la composición de la acronimia, aspecto que se abordará más adelante.

2 Aunque en ocasiones se recurre en publicidad a estrategias totalmente distintas, como las repeticiones o los pleonasmos. 


\section{¿CÓmo Se Define “PALABra”?}

En general, se habla holgadamente del concepto palabra, como un término ampliamente conocido. Sin embargo, es necesario, desde la lingüística, establecer una definición satisfactoria. Actualmente, la RAE la define como "segmento del discurso unificado habitualmente por el acento, el significado y pausas potenciales inicial y final" (DRAE 2001, s. v. palabra). A continuación se tratará de dilucidar la concepción de este término, sus límites y alcances. Pero antes de presentar una definición resulta conveniente realizar algunas precisiones teóricas. De acuerdo con los postulados de Martinet (1984), principalmente, se puede sostener que los morfemas o monemas $^{3}$ son las unidades gramaticales mínimas significativas, es decir, que no se pueden dividir en unidades significativas más pequeñas. A partir de aquí, es posible entender lo que el autor define por sintema: "una unidad significativa analizable formal y semánticamente en dos o más monemas, pero que sintácticamente mantiene con los otros elementos del enunciado las mismas relaciones que los monemas con los que alterna" (ibíd.: 289). Así, un sintema es una unidad lingüística que está constituida por dos o más monemas, aunque también puede estarlo por uno solo (como en césped), pero que funciona como un monema. En este sentido, se puede establecer la distinción entre este concepto y el de sintagma, que corresponde a la unión de dos o más monemas, pero que no funcionan como uno solo, definición que podría equipararse a la de frase en la gramática tradicional. Debido a lo anterior, se puede entender que Martinet sostenga que "adquieren también el estatus de sintema inmovilizaciones de sintagma" (ibíd.: 290), donde una inmovilización es una secuencia que se ha cristalizado en su significado debido a su alto valor funcional dentro de la lengua, pues "parece [...] que el transformarse en monema antes o después es el destino reservado de todo sintema de uso frecuente" (ibíd.). Entonces, resulta evidente que estrella de mar constituye un sintema, mientras que estrella de cine no, pues en este caso cine puede variar libremente en su paradigma por televisión, teatro, fútbol, etc. Teniendo en cuenta estas consideraciones, se puede llegar, siguiendo muy de cerca a Martinet, a la conclusión siguiente: palabra es un sintema autónomo constituido por uno o más morfemas que no se pueden separar. Luego, en caso de que esté constituido por más de una morfema de base, la palabra corresponde a la cristalización de un sintagma; así como en caso

\footnotetext{
3 Acá, se usarán indistintamente estos dos términos, aunque en algunos autores se pueden hallar diferencias terminológicas.
} 
contrario, en el otro extremo, son palabras también los morfemas como antes, sol, con, el, etc. ${ }^{4}$. Por lo tanto, se puede establecer que la palabra es la mínima unidad funcional, y no solo significativa, del lenguaje.

No está de más establecer en este punto una distinción entre lo que es la palabra gráfica y la palabra funcional. La primera corresponde a aquella cuyos constituyentes están gráficamente unidos y está, por lo tanto, delimitada por espacios en blanco, mientras que la palabra funcional corresponde a aquella que, independientemente de si se compone de una o más palabras gráficas, funciona como una sola palabra. Así, por ejemplo, de inmediato consta de dos palabras gráficas, pero actúa como una sola palabra funcional. Por supuesto, de acuerdo con los criterios antes señalados, en este estudio se entenderá palabra en su sentido funcional. Por lo tanto, puede establecerse que la palabra es "una unidad fonológica, una unidad de distribución, una unidad formal, una unidad semántica o de significado y una unidad funcional" (Fernández, Hervás y Báez 1984.: 45).

\section{PRECISIONES EN TORNO A DOS PROCESOS LEXICOGENÉSICOS: LA ACRONIMIA Y LA COMPOSICIÓN}

El caudal léxico de una lengua no constituye un número fijo, sino que en su devenir histórico va ampliando y reduciendo unidades. Este último fenómeno corresponde a los procesos denominados obsolescencia y mortandad léxicas, que consisten en un paulatino abandono del término, hasta que desaparece por completo del habla y termina por eliminarse de los diccionarios. De una riqueza y variedad mucho mayores son los procesos que incorporan nuevas unidades al sistema, ya que parece que la tendencia de los hablantes es hacia la creatividad orientada a la productividad, es decir, hacia buscar nuevas formas de expresión, las cuales se van fijando con el paso del tiempo. Estos procesos se agrupan en tres grandes grupos: creación ex nihilo (espontánea, sin antecedentes); vía externa (incorporación de unidades lingüísticas provenientes de lenguas extranjeras), y vía interna (formaciones al interior de una lengua).

4 Es importante recalcar en este punto que las preposiciones también se consideran monemas libres y, por lo tanto, palabras. 


\section{LA VÍA INTERNA COMO FUENTE DE CREACIÓN LÉXICA}

Consiste en tomar unidades disponibles en el sistema de la lengua y modificarlas a través de nuevas combinaciones para generar una palabra que no existía antes. También es posible hacer una distinción de base entre procesos que reducen elementos para generar una palabra más breve (acortamiento, abreviamiento y abreviatura) y procesos que alargan mediante adición de elementos (derivación, composición, parasíntesis y confijación).

\section{Acortamiento}

La acronimia responde al principio de la economía del lenguaje y se realiza a través de dos procesos: formación de siglas y de acrónimos, que reducen un grupo sintagmático (o frástico) a una sola palabra. Se trata de un procedimiento de creación léxica que reúne características tanto de la composición como del abreviamiento. Alvar (1996: 45) así lo expresa en su definición: "Un caso especial de abreviamiento es la acronimia, o unión del comienzo de una palabra con el final de otra, o, más raramente, el final de una y el comienzo de otra. Es una forma de composición muy moderna y de carácter técnico". La característica fundamental de todo acrónimo es que reduce a un solo lexema un sintagma, pero se agrega el hecho de que al menos uno de estos lexemas (o ambos) sufre(n) acortamiento interno (apócope o aféresis). De la cita anterior se desprenden las posibilidades de formación que puede tener un acrónimo, a saber:

1) reunir fragmentos de un grupo sintagmático en un solo lexema (el acrónimo), de lo cual se desprende que primero ocurren abreviamientos y después las partes restantes se reúnen en un nuevo lexema. Lo más frecuente es que la primera palabra del grupo sintagmático original sufra apócope, y la segunda, aféresis; aunque Alvar (1996) señala que también es posible que ocurra al revés, proceso que, por lo demás, no es infrecuente en el español de Chile. Por ejemplo, informática < información automática (donde información se abrevió por apócope; y automática, por aféresis).

2) resumir fragmentos de dos lexemas, aunque también puede abarcar tres o más, como ocurre con algunas organizaciones: SERNAM (Servicio Nacional de la Mujer), la que constituye la opción más frecuente.

Con respecto a la fragmentación de los componentes del acrónimo, esta puede ocurrir en todos ellos o solo en uno, aspecto que no consideran 
todos los autores. Casado (1999: 5087) avala esta idea: "también desde el punto de vista formal pueden distinguirse acrónimos en que sólo se fragmenta un elemento constituyente y acrónimos en que se fragmentan los dos. El primer caso es el más frecuente". De hecho, en la mayoría de sus ejemplos se aprecia abreviamiento de todos los componentes (como en secrefata< secretaria+azafata). Solo hay dos ejemplos en los que el segundo lexema se mantiene íntegro: docudrama (documental+drama) y cantautor (cantante+autor). Almela (1999: 206) define la acronimia como: "[...] la combinación, en un lexema nuevo, de un fragmento inicial de una unidad léxica y de un fragmento final de otra unidad léxica. [...] Las unidades combinables reducen previamente sus formas". De sus palabras se puede deducir que el acrónimo tiene un orden invariable en su constitución interna, el cual sería: primera unidad sufre apócope (como infor(mación)) y la segunda, aféresis (como (auto)mática), lo que daría el educto informática como un ejemplo clásico de acrónimo. Sin embargo, algunas páginas más adelante (ibíd.: 208) reelabora este aspecto del truncamiento y concluye que existen otras combinatorias, como palabra completa más fragmento (como dictablanda, publirreportaje o videófono). Si se considera el hecho de que el truncamiento puede ocurrir en un solo formante del acrónimo, vemos que este recurso es muy cercano a la composición y el único rasgo que diferencia ambos procedimientos es que en la composición se incluyen lexemas completos, con pequeñas variaciones en búsqueda de la eufonía (como en pelirrojo, donde $\mathrm{o}>\mathrm{i}$ ); mientras que en la acronimia, necesariamente, hay al menos un abreviamiento, pudiendo aparecer más. En este sentido resulta interesante rescatar la posición de Alvar (1996), quien incluye la acronimia dentro de la composición. En este trabajo se considerarán composición aquellos casos en los que los lexemas de base estén completos en su significante (salvo la adecuación eufónica descrita anteriormente) y acronimia cuando se aprecie abreviamiento (apócope o aféresis, incluso síncopa) en uno o en todos los lexemas formantes.

Con respecto a los ámbitos donde ha proliferado el uso de acrónimos, Lang $^{5}$ (1997: 259) señala que:

Tales formaciones resultan ser muy fugaces y proliferan formando parte de neologismos relacionados con el humor, consistente, precisamente, en la unión de forma imprevisible de partes de palabras. No obstante, puesto que constituyen un rasgo característico del lenguaje de la publicidad, la

5 Para respetar la terminología propia de cada autor, es conveniente aclarar que Lang (1997) utiliza acrónimo para referirse a lo que comúnmente llamamos sigla, y combinación (traducción de blend) para denominar a las uniones de lexemas con abreviamiento. 
empresa, el comercio y el periodismo se encuentran en el punto de mira del lenguaje del futuro. Como ocurría con los acrónimos, son útiles como respuesta a una mayor velocidad en la expresión que viene marcada por las exigencias actuales establecidas por los medios de comunicación de masas en general, suministrando fórmulas para titular concisamente todo tipo de instituciones. En este sentido, constituyen una de las más destacadas aportaciones lingüísticas al mundo de la avanzada tecnología.

Siguiendo a Lang (1997), en este estudio destacaremos que, debido a su gran poder de condensación, los acrónimos están sufriendo un proceso de revitalización en el español, proceso que va de la mano con el desarrollo de tres factores propios de la sociedad occidental de los siglos XX y XXI: el desarrollo de la publicidad (aspecto importante en sociedades que se orientan hacia consumo); el desarrollo de los medios de comunicación y, por último, la rapidez como valor determinante. En este sentido, el acrónimo da cuenta perfectamente de estos tres factores inherentes. Un enfoque más actual es el de Varela (2005), quien realiza un análisis pormenorizado de los acrónimos y los distingue de la composición de dos formas acortadas, límite que resulta sutil y a veces difícil de aplicar, como ella misma aclara: "en ciertos casos, no hay unanimidad en cómo analizar una creación léxica concreta: si como compuesto de dos formas acortadas o como 'acrónimo"” (ibíd.: 92).

\section{Alargamiento}

Tradicionalmente, la formación de palabras se ha estudiado dentro de la gramática, específicamente en la subdisciplina llamada morfología, bajo el nombre de lexicogenesia, pero, indudablemente, guarda una estrecha relación con la semántica lexemática, por lo cual se rescatarán aquí los aportes que ambas disciplinas ofrecen. La formación de palabras es un ámbito esencialmente creativo y no del todo normado, ya que las reglas que rigen su funcionamiento no están del todo explicadas y existen lagunas y preferencias por determinados afijos. En todo caso, sí es posible detectar en español algunas regularidades que permiten una descripción de la formación léxica. El principio básico es que se trata de una creatividad que forma nuevas unidades basándose en elementos previos; consiste en recombinar elementos ya presentes en el sistema de la lengua. En cuanto a la descripción de los recursos más importantes, encontramos cuatro procedimientos de formación de palabras principales: derivación, composición, parasíntesis y confijación. En el presente trabajo pondremos un énfasis especial en la composición, ya que es uno de los procesos que incide en el corpus de nuestra investigación. En términos generales, la composición consiste en la formación de una nueva palabra (entendida esta como unidad funcional), mediante la adición 
de dos o más unidades léxicas que pueden, además, funcionar por sí solas en otros enunciados. En términos de la gramática tradicional, la fórmula que resumiría la composición es: morfema de base + morfema de base, pudiendo existir entre ambos morfemas un elemento prepositivo. Ejemplos: secarropa, agridulce, guerra civil y caballito de mar, que corresponden, respectivamente, a un sustantivo, a un adjetivo, y a dos sustantivos. Los últimos dos ejemplos muestran casos en los que se mantiene un espacio gráfico entre las unidades formantes, constituyendo materia de discusión el grado de unión que puede darse en estos compuestos.

Alvar (1996) plantea una tipología de compuestos dependiendo del grado de unión gráfica que hay internamente, la que resulta interesante rescatar:

1) Sinapsia: participan usualmente tres unidades, siendo la segunda una preposición, por lo cual la primera unidad es el núcleo y la segunda, el determinante. Responde a casos como letra de cambio, olla a presión o caballo de batalla. La preposición de suele ser la más frecuente.

2) Disyunción: se considera que hay lexicalización porque los elementos del compuesto designan un solo referente, pero la preposición ha desaparecido y quedaron, por lo tanto, solo dos unidades. La primera tiene carácter nuclear, mientras que la segunda es una especificación. Se aplica a casos como cama nido, guerra civil o pez espada.

3) Contraposición: muestra un grado más elevado de unión entre los miembros del compuesto, ya que se escriben unidos por un guión, con lo cual cada uno mantiene su ortografía propia. Casos de compuestos por contraposición son: físico-químico y coche-cama.

4) Yuxtaposición: agrupa la mayor cantidad de casos. Se caracteriza porque "la fusión gráfica de los elementos participantes en el compuesto es total, así como su lexicalización y su gramaticalización. Estas formaciones son las que Bernard Pottier viene en llamar lexías compuestas" (Alvar 1996: 30). Por ejemplo, artimaña, menoscabar, aguardiente, cejïunto y cascanueces.

Lang (1997: 91) señala que: "el concepto de composición implica un estadio intermedio entre la palabra y la frase", principio con el que concordamos en nuestro trabajo. Se apoya en ejemplos conocidos del tipo cielorraso o enhorabuena, a los que denomina "compuestos ortográficos", debido a que muestran un grado semántico, funcional y visual de unión. Sin embargo, al igual que Alvar (1996), reconoce también la posibilidad de compuestos que no estén unidos gráficamente, pero que "constituyen una unidad semántica y mantienen relaciones sintácticas semejantes a las que presenta una estructura frástica u oracional" (ibíd.: 92). 
Un extenso estudio acerca de los procedimientos de formación de palabras en español lo realiza Ramón Almela (1999). Con respecto al origen del compuesto, dicho autor señala que suele invocarse a la oración como paráfrasis, pero no en todos los casos (ibíd.: 121):

No siempre está claro qué unidad lingüística se halla en el origen del compuesto, ni siquiera si existe tal origen. En las contadas ocasiones que pudiera haber acuerdo sobre tal origen, ¿no se le estaría confundiendo con una paráfrasis elaborada a posteriori? Dicha paráfrasis unas veces consistiría en un mero sintagma (hojalata $=$ hoja de lata), otras veces en una oración fácilmente recuperable (niño modelo $=$ niño que es digno de ser imitado) [...], mientras que en otras el enunciado parafrástico podría adoptar diversas formulaciones (hombre orquesta $=$ hombre que dirige una organización, que es necesario para un grupo, etc.) [...]

Resulta interesante en su planteamiento la inversión que hace con respecto al origen de la composición, ya que tradicionalmente se asume un sintagma u oración previos, del cual el compuesto sería una forma de condensación; sin embargo, para Almela, este fundamento puede no haber existido nunca. En este caso, la paráfrasis actúa como una interpretación del compuesto.

\section{Comparación entre acronimia y composición}

Tradicionalmente, en la gramática la acronimia y la composición se abordan por separado, considerando la primera como un proceso de reducción y la segunda como alargamiento, pero en realidad son muy similares, ya que la estructura básica de ambas es la unión en una sola unidad lexemática de dos o más palabras que además funcionan en lengua independientemente; con la salvedad de que en la acronimia una de las unidades (o todas) han sufrido un truncamiento de parte de su significante. Lo anterior se traduce, en casos concretos, en formas resultantes muy similares, como se aprecia en $a$. y $b$.:

\begin{tabular}{|l|}
\hline$a$. teléfonoayuda \\
b. fonoayuda \\
\hline
\end{tabular}

Mientras que $a$. se trata de una palabra compuesta (teléfono $+a y u d a), b$. es acrónimo solo porque la primera unidad (teléfono) sufrió aféresis, lo que la vuelve más breve y fácil de recordar. Al respecto, Lang (1997: 258) advierte sobre esta similitud: "sin embargo, si consideramos el hecho de que dos lexemas independientes se reúnen en uno solo, estas formaciones adquieren una característica fundamental que las relaciona con la composición". 


\section{EL ESTUDIO}

\section{CARACTERÍSTICAS DE LA INVESTIGACIÓN}

El corpus de nuestro estudio está constituido por 175 casos de palabras que incluyen formas innvadoras de composición o acronimia. Se presenta separado en dos grandes muestras:

a) el material sincrónico: corresponde a 102 casos obtenidos desde 2004 hasta 2007; incluyó la observación directa de carteles y letreros de edificios públicos, clínicas, paraderos de microbuses y estaciones de metro, además de la lectura de los periódicos La Tercera, El Mercurio y Las Últimas Noticias de los días sábado y domingo. También se recopilaron ejemplos de la televisión;

b) el material diacrónico: está formado por 73 casos extraídos desde la hemeroteca de la Biblioteca Nacional; incluyó la revisión de la primera quincena del mes de agosto de 1975,1980,1985,1990,1995 y 2000, en el caso del diario Las Últimas Noticias, y de 1975, 1980, 1985, 1990,1995 y 1998 y 2001 del diario La Tercera. El objetivo de este rastreo es detectar la presencia de formas similares a las halladas en el corpus sincrónico, para lograr establecer la época y el nivel de aparición del fenómeno detectado el año 2004.

Para ambos tipos de materiales, la presentación de los resultados se realizará a partir de la separación de dos criterios: el criterio referencial (que establece una relación entre la muestra y sus circunstancias contextuales) y el criterio lingüístico (que presenta el análisis morfológico, léxico y gramatical).

El criterio referencial se desglosa en las siguientes categorías:

a) Tipo de texto: se refiere a la fuente física desde donde se extrajo la muestra. Se separa en ocho grupos, los cuales son: páginas web, avisos publicitarios en diarios y revistas, boletas o facturas de empresas públicas y privadas, envases o sobres que envuelven un producto comercial, letreros públicos, avisos televisivos, entradas en guías telefónicas de la antigua CTC (Compañía de Teléfonos de Chile) y volantes o folletos publicitarios.

b) Fuente: entrega una breve referencia (espacial, institucional o contextual) que completa lo señalado en a).

c) Tipo de publicidad: se refiere a los dos tipos fundamentales: publicidad comercial y la institucional (llamada también propaganda). 
d) Referente: la clasificación alude al tipo de referente que tiene la muestra: si se trata de un objeto al cual denomina como nombre propio, o si se trata de algún servicio ofrecido por una institución.

Es necesario aclarar que en todos los casos se ha mantenido la tipología original, lo que implica respeto y mantención de: uso de mayúsculas; espacio separador de palabras; uso de negrita y/o cursiva; uso de colores y tamaños de letra.

El criterio lingüístico presenta cinco categorías de análisis:

a) Oración o frase subyacente: es una reconstrucción frástica y/u oracional que pretende mostrar, en un enunciado extendido (en el cual se restituyen verbos, preposiciones, cláusulas y otros elementos elididos) el mensaje de la expresión condensadora (acrónimo o compuesto).

b) Procedimiento de creación léxica: fundamentalmente se trata de composición o acronimia.

c) Clases de palabras involucradas: se recurre a las clases gramaticales de la gramática tradicional (sustantivo, adjetivo, adverbio, verbo, preposición).

d) Núcleo y determinantes: presenta cuál de los elementos es nuclear y cuál funciona como determinante, considerando el enunciado extendido.

e) Grado de unión gráfica de los elementos: hemos utilizado la terminología propuesta por Alvar (1996) para la composición, la cual se mantuvo para referirnos a los acrónimos, ya que se vio que no siempre forman una sola palabra gráfica (yuxtaposición).

\section{El MATERIAL SINCRÓNICO}

\subsection{Criterio referencial}

Un resumen de los aspectos extratextuales del corpus es el siguiente:

- Tipo de texto: ampliamente los letreros fijos tienen un alto número de representación $(27,7 \%)$, lo cual tiene relación con dos aspectos propios de este formato textual: primero, debido a que las letras son grandes, hay poco espacio para escribir, por lo cual la eficacia comunicativa de las pocas palabras presentes debe ser alta; segundo, los letreros, ubicados en lugares visibles y de paso, son de lectura rápida, otro aspecto que potencia la economía lingüística; los avisos comerciales (con una 
representación del 21,6\%) y las entradas en las guía telefónicas $(15,7 \%)$ tienen el mismo objetivo: captar rápidamente la atención del lector que tiene a su disposición, en la misma página, una gran cantidad de información; finalmente, categorías con menor representatividad son: páginas web $(9,8 \%)$; boletas y envases ( $7,8 \%$ cada una); letreros móviles $(3,9 \%)$; folletos $(2,9 \%)$; avisos televisivos $(1,9 \%)$ y los volantes $(0,9 \%)$.

- Tipo de publicidad: se aprecia una amplia mayoría de la publicidad comercial: $91,1 \%$ frente al 8,9\% de la publicidad institucional. Esta ventaja se explica por una razón simple: en nuestra sociedad hay mucha más publicidad con fines comerciales que con otros fines, por lo tanto, es más difícil hallar casos de propaganda, los cuales se remiten, en esta investigación, a unos pocos organismos del Estado (Ministerio de Justicia, MOP, Fundación Niño y Patria, entre otros).

- Referente: la mayoría corresponde a servicios que ofrecen empresas particulares o públicas (78\%), mientras que solo un $22 \%$ se trata de empresas, locales de comida u objetos que en su denominación exhiben una forma compuesta o acronímica, con lo cual estas últimas funcionan como sustantivos propios.

\subsection{CRiterio lingüístico}

\section{Las oraciones subyacentes}

Como se aprecia al leer la tabla, todos los casos recolectados, que corresponden a acrónimos o palabras compuestas, fueron susceptibles de reformularse en una oración, que expresa de manera menos condensada la misma información. Al contrastar los casos de la muestra con sus correspondientes oraciones o frases subyacentes se demuestra que en estas últimas:

- La forma de entregar la información es más extensa, por lo cual, lógicamente hay más palabras y se entregan más detalles.

- La información que no aparece en superficie se deduce del contexto en que aparecen estas palabras y del conocimiento de mundo del hablante, lo cual deja abierta la posibilidad de que haya formas ambiguas o poco explícitas.

- Se establecen relaciones de determinación internas, siendo la más frecuente un núcleo sustantivo con un complemento del sustantivo, como en: 
Ejemplos con complemento del sustantivo:

- servipag = servicio para realizar pagos

- fono pago = teléfono para realizar pagos

- BancoEstado = Banco del Estado

- fotoborde $=$ borde para fotografías

- fonoservicio $=$ teléfono para acceder a un servicio de determinada empresa.

Hay algunos ejemplos en que se percibe una oración subyacente un poco más compleja, que al hacerse explícita requiere de una cláusula adjetiva:

Ejemplos con cláusula adjetiva:

- Telemergencia: emergencia que se resuelve a través del teléfono

- TELEPEAJE: peaje carretero que se paga a través del teléfono

- AutoPlaza: lugar (plaza) donde se venden automóviles

Los casos vistos anteriormente corresponden a oraciones unimembres con un núcleo nominal y son la mayoría de la muestra: 99 casos (97\%). Evidentemente, se trata de condensaciones, que a veces sintetizan una gran cantidad de información, como en:

- Instanparis: servicio instantáneo de información sobre cuenta de Almacenes Paris

- Fonomujer: teléfono para que llamen las mujeres y se informen sobre el método anticonceptivo publicitado.

También puede ocurrir que la oración subyacente sea breve, como en Econópticas, que se entiende, simplemente, como "óptica económica".

Solo en tres casos $(2,9 \%)$ encontramos predicados verbales que dan a la oración subyacente el carácter de bimembre verbal, a saber:

- SOLODIVORCIOS: (estudio jurídico) que sólo tramita divorcios

- ChileInventa: Chile inventa

- ENTRELATAS: pub donde los clientes están entre latas de cerveza.

Estos análisis parten del supuesto de que la oración subyacente presentada es una expresión adecuada del acrónimo o el compuesto; pero ello no impide que estos puedan reformularse de manera distinta y generar así, diferentes elementos subyacentes. Si contrastamos la extensión de los elementos subyacentes con la síntesis de los casos de la muestra, puede concluirse que no solo se eliden preposiciones, sino también palabras (como infinitivos) 
y diversos complementos. Debido a la supresión de palabras lexemáticas y preposiciones, la forma compuesta o acronímica resultante puede llegar a ser ambigua. Para su interpretación se requiere de elementos contextuales, como se dijo. En el caso de las múltiples formaciones con fono destaca el hecho de que diversos acrónimos que lo incluyen no siempre involucran la misma relación semántica, como se aprecia en estos ejemplos:

Tabla 1: interpretaciones de acrónimos

\begin{tabular}{|l|l|}
\hline Caso de la muestra & Interpretación \\
\hline 1. FONO PIZZA & Teléfono para pedir pizza a domicilio \\
\hline 2. FONO DROGA & $\begin{array}{l}\text { Teléfono para hacer denuncia sobre consumo } \\
\text { o tráfico de drogas }\end{array}$ \\
\hline 3. FONORATAS & $\begin{array}{l}\text { Teléfono al cual se puede llamar para solucionar } \\
\text { problemas de ratas }\end{array}$ \\
\hline 4. FONO NIÑOS & Teléfono para denunciar maltrato infantil \\
\hline
\end{tabular}

Es necesario recalcar el hecho de que la oración subyacente a las formaciones analizadas requiere de una interpretación muy dependiente del conocimiento contextual extralingüístico. Si así no fuera, se podrían realizar interpretaciones erróneas, tales como pensar que FONO DROGA remite a un teléfono para pedir drogas; o FONO NIÑOS es un teléfono para que los niños busquen entretención, por analogía con FONO PIZZA O FONO TAXI, analogía que resulta ser falsa, ya que cada una de las formaciones implica un objetivo distinto. En los ejemplos se aprecia que, desde un punto de vista de caso semántico, (telé)fono actúa como instrumento que físicamente permite realizar una acción, que en todos los casos implica contactarse con una empresa o institución, pero es pertinente aclarar que la naturaleza de dicha acción es distinta, ya que implica propósitos distintos, como se presenta en la siguiente tabla:

Tabla 2: Propósitos asociados al uso de fono-

\begin{tabular}{|l|l|c|}
\hline Propósito & Muestras & Total \\
\hline 1. Denunciar & $\begin{array}{l}\text { FONO NIÑOS, FONO DROGAS, FONO } \\
\text { DENUNCIA }\end{array}$ & 3 \\
\hline 2. Pagar & FONO PAGO & 1 \\
\hline 3. Comprar & $\begin{array}{l}\text { FonoCompras, Fono Compras, } \\
\text { FONOCOMPRAS }\end{array}$ & 3 \\
\hline
\end{tabular}




\begin{tabular}{|c|c|c|}
\hline 4. Vender & $\begin{array}{l}\text { FONO VENTAS, Fono ventas, Fonos } \\
\text { Ventas }\end{array}$ & 3 \\
\hline 5. Consultar & $\begin{array}{l}\text { FonoPresto, Fonoconsulta, Fono Consul- } \\
\text { ta(2), Fono Consultas, FONO CONSULTA, } \\
\text { FONO CAMINO, FONOTAG }\end{array}$ & 8 \\
\hline 6. Informarse & Fonomujer, FONOSIDA & 2 \\
\hline $\begin{array}{l}\text { 7. Acceder a servicios } \\
\text { de diverso tipo }\end{array}$ & $\begin{array}{l}\text { FonoServicio (3), fonoServicio, FONO } \\
\text { SERVICIO, Fono Servicio }\end{array}$ & 6 \\
\hline $\begin{array}{l}\text { 8. Contactarse con una } \\
\text { empresa, con cual- } \\
\text { quier fin }\end{array}$ & $\begin{array}{l}\text { Provifono, FONOCENTRAL, Fono Salo, } \\
\text { FonolANSA, INSTANFONO }\end{array}$ & 5 \\
\hline $\begin{array}{l}\text { 9. Reservar horas } \\
\text { médicas }\end{array}$ & Fonoreserva(2), FONO RESERVA & 3 \\
\hline 10. Reclamar & FONO RECLAMO & 1 \\
\hline $\begin{array}{l}\text { 11. Llamar en caso de } \\
\text { emergencia }\end{array}$ & FONO EMERGENCIA & 1 \\
\hline 12. Pedir ayuda & Fono Ayuda, FONO AYUDA & 2 \\
\hline $\begin{array}{l}\text { 13. De uso gratuito } \\
\text { (para quien llama) }\end{array}$ & Fono Gratis 24 hrs. & 1 \\
\hline $\begin{array}{l}\text { 14. Llamar cuando se } \\
\text { es cliente }\end{array}$ & $\begin{array}{l}\text { FONOCLIENTE, FONO CLIENTE, Fo- } \\
\text { nocliente }\end{array}$ & 3 \\
\hline $\begin{array}{l}\text { 15. Adquirir un } \\
\text { producto (o servicio) } \\
\text { específico de una } \\
\text { empresa }\end{array}$ & $\begin{array}{l}\text { Fono Taxi(7), Fono taxi, Fonotaxi(4), } \\
\text { Fonotaxis, Fono Taxis, FONO } \\
\text { PIZZA, Fonoviajes, FONOVIAJES, } \\
\text { FONOTIMBRE, FONORATAS, FONO } \\
\text { SOUR, Fono reparto }\end{array}$ & 21 \\
\hline $\begin{array}{l}\text { 16. Hacer una ofrenda } \\
\text { religiosa }\end{array}$ & FONO OFRENDA & 1 \\
\hline $\begin{array}{l}\text { 17. Llamar a una cen- } \\
\text { tral telefónica }\end{array}$ & Fonocenter & 1 \\
\hline 18. Aplacar la sed & Fono sed & 1 \\
\hline TOTAL & & 63 \\
\hline
\end{tabular}

Sobre la tabla 2, podemos establecer que:

- con respecto a los propósitos 5 y 6 (consultar e informarse, respectivamente), nos parece que aparecen como criterios distintos, aunque pueden 
parecer sinónimos, ya que el primero implica una petición breve de información; en tanto que informarse se entiende como una petición de información más compleja, sobre características de un producto y conductas a seguir. Recordemos que este último se relaciona con un nuevo método anticonceptivo y con formas de prevención del SIDA;

- resulta interesante una comparación entre dos formas como Fono Compras y Fono ventas, ya que aluden al mismo referente: la acción que consiste en que alguien llama por teléfono para comprar un producto. Solo cambia la perspectiva con que se enfoca el proceso. La primera (Fono Compras) focaliza al cliente (el que "compra", por lo tanto, el número telefónico que se da es para este compre); la segunda, a la empresa que posee un teléfono especial para realizar ventas (en cuyo caso se entiende que en el teléfono se realiza la venta);

- en la mayoría de los casos, la relación entre los dos términos constituyentes es bastante directa, como se aprecia en FONO EMERGENCIA (teléfono al cual recurrir en caso de emergencia) y otros. Llaman la atención tres casos en los que no se da una relación tan directa. Se trata de Fono sed, FonoPresto e Instanfono, los cuales pasaremos a analizar a continuación:

* Fono sed: debería haber figurado en la categoría 4. (Vender) ya que se trata de un teléfono de la empresa Cascadas, que vende bidones de agua soda. El verdadero sentido no es tan amplio como podría pensarse, ya que plantea que la sed, en su totalidad, se aplaca únicamente con ese producto. Una formación más directa habría sido *Fono soda $\mathrm{o}$ *Fono Cascadas $^{6}$.

* FonoPresto: el constituyente Presto aquí no funciona como palabra lexemática, ya que corresponde a un nombre propio: el de la tarjeta de crédito otorgada por el supermercado Líder. Por lo tanto, una interpretación válida, pero no totalmente acertada, sería que es un teléfono "de respuesta rápida (presta)", o que quienes lo atienden están "prestos" a resolver cualquier problema. Como se trata de publicidad comercial, no se descarta que el nombre elegido para la tarjeta busque intencionalmente una relación semántica con el adjetivo homógrafo.

* Instanfono: corresponde a un teléfono de la tienda Almacenes Paris, la que privilegia la idea de rapidez (instan) frente a los problemas

${ }^{6}$ Hemos usado el asterisco (*) para indicar formas hasta ahora inexistentes en la realidad del español de Chile. 
que puedan plantear los clientes, que el nombre de la empresa (como sería

* Fono Paris) o una forma socialmente más difundida (fonoconsulta, por ejemplo).

\section{Procedimientos de creación léxica}

Tabla 3: resumen de procedimientos de creación léxica

\begin{tabular}{|l|c|c|}
\hline Procedimiento & Número de casos & Porcentaje \\
\hline Acronimia & 86 & 84,3 \\
\hline Composición & 16 & 15,7 \\
\hline Total & $\mathbf{1 0 2}$ & $\mathbf{1 0 0 \%}$ \\
\hline
\end{tabular}

La tabla 3 muestra que, contrariamente a lo planteado en el comienzo de este estudio, no es la composición el procedimiento más frecuente, ya que esta alcanza un $15,7 \%$ de representatividad, en contraste con el $84,3 \%$ que exhibe la acronimia. Esto se debe al hecho de que los acortamientos ocurren con asombrosa frecuencia, por lo tanto, en la unión de la palabras con supresión de preposiciones es muy común que además haya aféresis o apócopes (no se registra ningún caso de síncopa). Los casos en que los morfemas de base se conservan completos (casos de composición) se mostraron exiguos. La situación anterior muestra una marcada tendencia a la economía del lenguaje en el discurso publicitario, ya que no solo aparecen palabras compuestas, las que en sí contienen una información susceptible de expresarse por medio de frases u oraciones; sino que, añadido a esto, muchas palabras formantes están acortadas. Por consiguiente, la tarea de leer el mensaje y asignarle la coherencia necesaria corresponde al receptor. Un caso prototípico de acrónimo es lo que ocurrió con la forma teléfono, originalmente un confijado formado a partir de dos afijos de origen clásico (tele, "lejos" y fono, "sonido"), del cual pervive fono ya no con el valor afijal, sino como la forma acortada (mediante aféresis) de teléfono. Así, un hablante de español de Chile del siglo XXI, al ver una forma como fonoreclamo o fonopago entiende que se trata de una acción realizada a través del teléfono. Escapa a esta constante el caso Telepizza, porque se mantuvo la base tele. 
Tipos de acortamiento en los casos de acronimia

Tabla 4: acortamientos en acrónimos

\begin{tabular}{|l|c|c|}
\hline \multicolumn{1}{|c|}{ Acortamiento } & Número de casos & Porcentaje \\
\hline Aféresis + palabra completa & 67 & 77,9 \\
\hline Apócope + palabra completa & 8 & 9,3 \\
\hline Palabra completa + apócope & 4 & 4,6 \\
\hline Apócope + aféresis & 3 & 3,5 \\
\hline Aféresis + apócope & 2 & 2,3 \\
\hline Sigla + aféresis & 1 & 1,2 \\
\hline Aféresis + sigla & 1 & 1,2 \\
\hline Total & $\mathbf{8 6}$ & $\mathbf{1 0 0 \%}$ \\
\hline
\end{tabular}

La mayoría de los casos recogidos muestra que la tendencia más frecuente en el español de Chile actual, que se refleja en la publicidad escrita, es la acronimia que involucra una palabra completa, lo que contradice a Almela (1999: 206 y 207), quien la define en función del truncamiento de todas las unidades: "La acronimia puede definirse como la combinación, en un lexema nuevo, de un fragmento inicial de una unidad léxica y de un fragmento final de otra unidad léxica. [...] la primera unidad sufre apócope, y la segunda, aféresis". Como señalamos en el marco teórico, Casado (1999: 5087), contempla la posibilidad de que haya en el acrónimo una palabra completa. Vistos nuestros casos, claramente las definiciones en la línea de Almela resultan insuficientes para describir la acronimia, ya que en un $91,8 \%$ de los casos el acrónimo trunca un solo formante y deja intacto el otro; mientras que solo cinco casos $(5,8 \%)$ responden a la fórmula de acortamiento en ambas unidades léxicas. La fórmula dada por Almela (apócope+aféresis) solo aparece en tres casos $(3,5 \%)$. Resulta curioso el hecho de que dos casos $(2,4 \%)$ involucren siglas, lo cual implica dos procesos consecutivos de acortamiento: primero en la constitución de una sigla, y después, en esta sigla que se une con un fragmento de otra palabra para conformar el acrónimo; proceso que requiere de un receptor bastante activo para realizar la reconstitución del sentido. Los casos citados corresponden a fonoIANSA y Pucmático, que pueden entenderse así:

- fonoIANSA: teléfono para comunicarse con la empresa IANSA ( $<$ Industria Azucarera Nacional Sociedad Anónima) 
- Pucmático: sistema automático de inscripción de cursos para alumnos de la PUC (Pontificia Universidad Católica).

\section{Clases de palabras presentes en la muestra}

En este apartado se fija la atención en las categorías gramaticales presentes y reconocidas por la gramática tradicional, a saber: sustantivo, adjetivo, verbo, adverbio, preposición, relativo, subjunción e interjección. Cabe aquí aclarar que no se ha considerado como criterio de análisis el orden en que aparecen los constituyentes, por lo cual una formación sustantivo + adjetivo figura junto a adjetivo + sustantivo.

Tabla 5: Clases de palabras

\begin{tabular}{|c|c|c|}
\hline Clases de palabra & Número de casos & Porcentaje \\
\hline Sustantivo + sustantivo & 85 & 83,4 \\
\hline Adjetivo + sustantivo & 10 & 9,8 \\
\hline Sustantivo + adverbio & 3 & 2,9 \\
\hline Verbo + sustantivo & 2 & 2,1 \\
\hline Verbo + adverbio & 1 & 0,9 \\
\hline Preposición + sustantivo & 1 & 0,9 \\
\hline Total & 102 & $100 \%$ \\
\hline
\end{tabular}

En términos generales, se observa que los sustantivos aparecen como favoritos en los acrónimos y las palabras compuestas, ya que participan prácticamente de todos los casos de la muestra. Se exceptúa únicamente el envase de Nestlé llamado sirvefácil, porque corresponde a la unión de verbo y adverbio. Los morfemas sustantivos unidos entre sí son el 83,4\% de todos los casos, lo cual marca una alta frecuencia que separa con gran ventaja numérica a este tipo de unión de las demás clases de palabras. Solo la unión de adjetivo + sustantivo tiene un cierto grado de representatividad $(9,8 \%)$; mientras adverbio + sustantivo alcanza un $2,9 \%$; verbo+sustantivo, un $2,1 \%$; y preposición + sustantivo solo tiene un caso, con un $0,9 \%$, al igual que verbo+adverbio. Desde otro punto de vista, casi en la totalidad de los casos, la forma compuesta o acronímica está constituida por bases propias del español. Solo cuatro casos $(3,9 \%)$ corresponden a extranjerismos: se trata de los anglicismos bank (en BankBoston), center (Fonocenter), stickers (FOTOSTICKERS) y ticket (Feriaticket). El resto (96,1\%) son lexías patrimoniales del español o bien adaptaciones. 
Unión gráfica entre los componentes

Tabla 6 : grados de unión gráfica

\begin{tabular}{|l|c|c|}
\hline Grado de unión & Número de casos & Porcentaje \\
\hline Yuxtaposición & 64 & 62,7 \\
\hline Disyunción & 38 & 37,3 \\
\hline Total & $\mathbf{1 0 2}$ & $\mathbf{1 0 0 \%}$ \\
\hline
\end{tabular}

Como era de esperarse, la yuxtaposición, es decir, la formación de una nueva palabra a partir de elementos preexistentes sin separación gráfica entre estos tiene una amplia representatividad, ya que abarca el $62,7 \%$ de los casos. La disyunción es la forma preferida en segundo lugar, con un $37,3 \%$ de ocurrencias. La contraposición, que marca la relación entre los formantes con un guión, no está representada, lo que evidencia que para los hablantes el guión terminó sintiéndose como un signo innecesario y, quizás, poco cercano. Resulta interesante mostrar que hay formas exactamente iguales en su escritura, salvo por el blanco intermedio, como Fono consulta y Fonoconsulta. No se perciben diferencias de sentido entre formas con disyunción y formas con yuxtaposición.

A continuación, se presentan separados algunos aspectos relevantes:

Vacilaciones en la escritura

Tabla 7: alternancia del espacio intermedio

\begin{tabular}{|l|l|}
\hline \multicolumn{1}{|c|}{ Caso } & \multicolumn{1}{c|}{ Referencia } \\
\hline $\begin{array}{l}\text { 1) Fono Ayuda } \\
\text { FONO AYUDA }\end{array}$ & (www.inp.cl) \\
(Agrupación AVAT)
\end{tabular}




\begin{tabular}{|c|c|}
\hline $\begin{array}{l}\text { 4) Fono Consulta } \\
\text { Fono consulta } \\
\text { Fono Consultas } \\
\text { Fonoconsulta } \\
\text { FONO CONSULTA }\end{array}$ & $\begin{array}{l}\text { (cartulinas Proarte) } \\
\text { (letrero Reforma Procesal Penal) } \\
\text { (www.aquipropiedades.cl) } \\
\text { (guía comercial CTC) } \\
\text { (Metro de Santiago) }\end{array}$ \\
\hline $\begin{array}{l}\text { 5) Fonoreserva } \\
\text { Fonoreserva } \\
\text { FONO RESERVA }\end{array}$ & $\begin{array}{l}\text { (Clínica Dávila, antigua Urgencia) (Clínica Vida } \\
\text { Integra) } \\
\text { (Clínica Dávila, nuevo centro médico) }\end{array}$ \\
\hline $\begin{array}{l}\text { 6) FONO SERVICIO } \\
\text { FonoServicio } \\
\text { FonoServicio } \\
\text { FonoServicio } \\
\text { Fono Servicio } \\
\text { FonoServicio } \\
\end{array}$ & $\begin{array}{l}\text { (Clínica Dávila, nuevo centro médico) } \\
\text { (Clínica Dávila, antigua Urgencia) } \\
\text { (boleta de Aguas Andinas) } \\
\text { (boleta de farmacia Cruz Verde) } \\
\text { (boleta de farmacia Cruz Verde) } \\
\text { (boleta de Chilectra) }\end{array}$ \\
\hline $\begin{array}{l}\text { 7) Fonoviajes } \\
\text { FONOVIAJES }\end{array}$ & $\begin{array}{l}\text { (catálogo de tienda Johnson's) } \\
\text { (catálogo de tienda Johnson's) }\end{array}$ \\
\hline $\begin{array}{l}\text { 8) Fono Taxi (7) } \\
\text { Fonotaxi (4) } \\
\text { Fono taxi } \\
\text { Fonotaxis } \\
\text { Fono Taxis }\end{array}$ & (todos en guía comercial de CTC) \\
\hline $\begin{array}{l}\text { 9) FONO VENTAS } \\
\text { FONO VENTAS } \\
\text { Fono Ventas } \\
\text { Fonos Ventas }\end{array}$ & $\begin{array}{l}\text { (letrero de Nissan Cidef) } \\
\text { (Inmobiliaria IMSA) } \\
\text { (www.libreriaOlejnik.cl) } \\
\text { (www.libreriaOlejnik.cl) }\end{array}$ \\
\hline
\end{tabular}

Encontramos nueve casos en los cuales aparece la misma formación, pero con sutiles variaciones en la escritura. Todos los casos corresponden a acrónimos iniciados con el segmento fono-. Estas variaciones se manifiestan a través de diversos recursos gráficos, como existencia o inexistencia de espacio gráfico entre formantes; uso variable de mayúscula (a veces en toda la palabra, otras solo en la primera letra y, por último, mayúscula dentro del acrónimo); negrita; cursiva y colores. Este último recurso es poco productivo y se observa en FonoServicio (boleta de Chilectra). Las diferencias gráficas no 
involucran, obviamente, diferencias en la pronunciación. Pero como nuestro corpus es de textos escritos, estas diferencias muestran que en los hablantes no se ha fijado todavía una manera estandarizada de escribir los acrónimos. Hay una tendencia a diferenciar visualmente a los constituyentes de la nueva formación, como se aprecia en FONOVIAJES y otros casos que no figuran aquí porque tienen una forma única de escritura (Prontofertas, por ejemplo). La escritura con disyunción se revela más cercana a la oración subyacente, ya que mantiene los elementos formantes con independencia gráfica. Pero esta tendencia es contraria a la definición misma de acrónimo, que reúne en una misma palabra (entendiéndose por "misma" una palabra gráfica) segmentos de otras. Por lo tanto, se acerca más a un tipo de composición, con lo cual surgen dudas en tomo a los límites precisos entre composición y acronimia. En la tabla presentada hay 33 muestras, de las cuales 15 (el 45,4\%) corresponden a formas yuxtapuestas; y $18(54,6 \%)$, a formas disyuntivas, es decir, la mayoría. La tendencia es a la separación gráfica, es decir, los hablantes sienten que el acrónimo todavía no es totalmente una unidad semántica, funcional y visual.

\section{Casos de yuxtaposición y mayúscula en el segundo constituyente}

En el apartado anterior se concluyó que la tendencia general es a generar acrónimos (y palabras compuestas, agregamos ahora) que gráficamente no son una unidad, de modo que el blanco intermedio mantiene, en cierta medida, la autonomía de cada lexema participante y, así, su origen frástico. Además de esta posibilidad de separación de los formantes, existe la forma yuxtapuesta, que es el grado máximo de fusión. Pero hay un estado intermedio que se caracteriza por la unión gráfica de los elementos, pero mantiene la mayúscula para el segundo de estos. Se trata de los diez casos que se presentan a continuación, donde se aprecia que el segundo constituyente, a pesar de estar unido al primero, mantiene la mayúscula, escritura que va derechamente en contra de la tradición de nuestro idioma, que desde siempre ha reservado el uso de mayúscula solo a la primera letra de una palabra (cf. Real Academia Española. 1999. Ortografia de la lengua española. Madrid: Espasa. Capítulo III):
1. Chilelnventa
2. AutoPlaza
3. BancoEstado
4. BankBoston
5. FonoServicio (3)
6. FonoCompras 7. FonoPresto
8. RedCompra
9. SuperHipoteca
10. FonoIANSA 
Se trata, por tanto, de un uso antiortográfico, si lo vemos desde un punto de vista normativo, pero novedoso y funcional, si pensamos en el hablante que está emitiendo un texto escrito, de carácter público, y que tiene dudas acerca de estas palabras "nuevas", que no forman parte de la tradición idiomática, pero que le son altamente útiles.

\section{Casos de yuxtaposición donde no hay duplicación de " $r$ "}

Otro interesante aspecto que sigue la huella del apartado anterior es el de las formaciones que en su escritura no presentan duplicación de "r" entre dos vocales, siendo esto una exigencia de nuestra lengua, para expresar gráficamente el fonema multivibrante. Se trata de:

\section{Previred 2. FOTORETOQUE \\ 3. Fonoreserva \\ 4. Metrored}

Son cuatro casos que constituyen el 3,9\% de toda la muestra. Si bien no es un porcentaje alto, muestra que la escritura de formaciones compuestas y acronímicas no es homogénea entre los hablantes y presenta hechos que podríamos calificar como incorrecciones, según un criterio normativo. Desde un punto de vista funcional, estas "faltas" no alteran la comprensión ni la eficacia comunicativa del texto.

\section{Vacilaciones en el mismo texto}

El último aspecto por analizar remite a dos casos en los que hay una escritura diferente de la misma forma, en el mismo texto y con apenas unas líneas de separación. Se trata de:

Tabla 8: escritura diferente en el mismo texto

\begin{tabular}{|l|l|}
\hline \multicolumn{1}{|c|}{ Muestra } & \multicolumn{1}{c|}{ Fuente } \\
\hline 1) Fono Servicio y Fonoservicio & Boleta de Farmacia Cruz Verde \\
\hline 2) Fonoviajes y FONOVIAJES & Catálogo de tienda Johnson 's \\
\hline
\end{tabular}

A esto se agrega la boleta de pago de Aguas Andinas, donde ocurre lo mismo, pero con las formas FONO PAGO y Fonoservicio. Estos tres casos refuerzan la afirmación hecha en el sentido de que no se ha fijado en la escritura una manera de graficar los acrónimos. 


\section{El MATERIAL DiACRÓNICO}

\subsection{Criterio referencial}

Es necesario realizar dos precisiones en este apartado. La primera es que todas las muestras corresponden a avisos publicitarios, en cuanto al tipo de texto, por lo que esta información se omitirá en la tabla. La segunda precisión es que hay muestras que aparecen repetidas en los años consultados, pero en distintas fechas de aparición. Se ha optado por mantener todas las apariciones con la finalidad de que se note cómo varía realmente el fenómeno a lo largo del tiempo.

Tabla 9: total de muestras por año

\begin{tabular}{|l|c|c|}
\hline Año & Número de casos & Porcentaje \\
\hline 1975 & 3 & 4,1 \\
\hline 1980 & 5 & 6,9 \\
\hline 1985 & 14 & 19,1 \\
\hline 1990 & 6 & 8,3 \\
\hline 1995 & 21 & 28,8 \\
\hline $1998-2001$ & 24 & 32,8 \\
\hline TOTAL & $\mathbf{7 3}$ & $\mathbf{1 0 0 \%}$ \\
\hline
\end{tabular}

Las muestras evidencian un aumento sostenido desde el año 1975, con una baja en 1990, la cual no es posible explicar aquí, porque no hemos hecho un estudio contextual ni discursivo de la prensa escrita chilena en esa década. De todas maneras, se observa una tendencia creciente, ya que de un tímido $4,1 \%$ del total de casos en 1975 , se llega a porcentajes cercanos al $30 \%$ en los segmentos más cercanos al presente: 1995 y 1998-2001. El descenso observado en 1990 ( $8,3 \%$ de casos) es solo en relación con el tramo anterior: en 1985 hay catorce casos (con una representatividad del 19,1\%); porque igual se marca un aumento si comparamos ese año 1990 con 1980 (6,9\%) y el citado 1975. El más alto porcentaje de casos es en el último tramo (1998-2001, con un 32,8\%), es decir, a medida que se avanza en años, sube el número de casos. Los datos antes citados reflejan un aumento en el uso de formas compuestas y acronímicas en los últimos treinta años, en textos escritos de carácter público. En cuanto al referente, los datos dejan ver que la mayoría de las muestras $(78,1 \%)$ corresponden a nombres propios de 
empresas que pertenecen a rubros tan variados como bancos, financieras, servicios automotores, parques de diversiones, telecomunicaciones y otros. Esta situación demuestra que los procesos acronímicos y de composición son mucho más vitales para dar nombre a una institución que para nominar servicios ofrecidos por empresas, ya que este último uso corresponde al $21,9 \%$ de los casos.

\subsection{Criterio lingüístico}

Vista la cantidad de muestras por cada fecha, no resulta necesario mostrar las repetidas. Hecha esta operación, el corpus se redujo a 60 casos.

\section{Las oraciones subyacentes}

Se puede apreciar cómo, en general, es posible, en las muestras de esta sección, hacer una reconstrucción de una frase u oración subyacente en ellas. Sin embargo, en algunas ocasiones, esta reconstrucción resulta al menos difícil, pues no se aprecia una clara relación entre los componentes de la muestra. En algunos casos, incluso, la formación resultante implica una mera yuxtaposición de términos, sin relación semántica, como sucede con CASAROYAL o Entelchile. En síntesis, se observa en el corpus diacrónico una menor complejidad que la hallada en el corpus sincrónico en cuanto a la reconstrucción de la frase u oración subyacente, pues en el primero abundan más las frases, a modo de un sintagma nominal, y no las oraciones; y además, en muchos casos la formación implica solamente la unión de un sustantivo con un adjetivo, como en FACILPLAN, MUNDOMÁGICO, donde el adjetivo indica no una predicación, sino solo una atribución del sustantivo. Cabe destacar aquí el caso de Construmart, ya que no fue posible dar cuenta de la oración subyacente, debido a que no está claro el aporte semántico de la partícula mart.

\section{El caso de fono}

La utilización de la partícula fono es muy baja en el corpus diacrónico. Solo se rastrea un caso: Fono-crédito, el cual tiene la particularidad de ser una contraposición desde el punto de vista de la unión gráfica (uso de guión). Así, se deduce que en la época que abarca este corpus, aún no se hace funcional la partícula fono para las composiciones o acronimias. El caso que más se asemeja al uso de fono en el corpus sincrónico es el de INFODROGAS, que posee una significación casi idéntica al caso FONO $D R O G A$ del corpus sincrónico. 
Procedimientos de creación léxica

Tabla 10: síntesis de procedimientos de creación léxica

\begin{tabular}{|l|c|c|}
\hline Procedimiento & Número de casos & Porcentaje \\
\hline Acronimia & 32 & 53,3 \\
\hline Composición & 26 & 43,3 \\
\hline Confijación & 1 & 1,7 \\
\hline Derivación & 1 & 1,7 \\
\hline Total & $\mathbf{6 0}$ & $\mathbf{1 0 0 \%}$ \\
\hline
\end{tabular}

En la tabla precedente se observa que los procesos lexicogenésicos más fuertemente involucrados son, al igual que en el corpus sincrónico, la composición y la acronimia. Solamente existe un caso, y por lo tanto marginal, de derivación y otro de confijación, si consideramos que tanto super como extra son afijos de tipo prefijos que se unieron en el adjetivo superextra. La acronimia encabeza las preferencias de uso, con un 53,3\% del total de los casos. No obstante, los casos de composición forman un número considerable, seguido a poca distancia de la acronimia, con un $43,3 \%$. De este modo, el uso de la composición no se encuentra cuantitativamente tan alejado del de la forma más económica encontrada, la acronimia, ya que ambos se acercan al 50\%.

Unión gráfica entre los componentes

Tabla 11: grado de unión gráfica

\begin{tabular}{|l|c|c|}
\hline Procedimiento & Número de casos & Porcentaje \\
\hline Yuxtaposición & 50 & 83,3 \\
\hline Contraposición & 7 & 11,7 \\
\hline Disyunción & 3 & 5,0 \\
\hline Total & $\mathbf{6 0}$ & $\mathbf{1 0 0}$ \\
\hline
\end{tabular}

De acuerdo con la tabla 11, se puede decir que la forma gráfica dominante entre los componentes de cada formación analizada es la yuxtaposición, que 
acapara el 83,3\% de los casos. En el otro extremo se encuentra la disyunción con solamente un 5\%. Por último, y como punto interesante, se aprecia que la contraposición tiene una representatividad del $11,7 \%$, lo cual es destacable en comparación con la nula presencia en el corpus sincrónico. Esto quiere decir que en esta época aún es necesario para los hablantes marcar la separación, a través del guión, entre los términos que forman el compuesto o el acrónimo.

\section{Análisis comparativo entre el corpus sincrónico y el corpus diacrónico}

La oración subyacente

Una lectura de los elementos subyacentes muestra una mayor complejidad en el corpus sincrónico, ya que se perciben más unidades elididas y relaciones de recursividad más densas que en el diacrónico, en el cual abundan sintagmas, la mayor parte nominales, con relaciones internas más simples. Por ejemplo, Electromat implica dos constituyentes y la relación entre ambos es "materiales electrónicos", un sintagma nominal de núcleo materiales. Provimático del corpus sincrónico también tiene dos elementos, pero la relación entre estos es más compleja: "servicio automático para obtener cartola de AFP Provida".

\section{Procedimientos de creación léxica}

Tabla 12: procedimientos de creación léxica

\begin{tabular}{|l|l|l|l|}
\hline \multicolumn{2}{|c|}{ C. sincrónico } & \multicolumn{2}{c|}{ C. diacrónico } \\
\hline Acronimia & $84,3 \%$ & Acronimia & $53,3 \%$ \\
\hline Composición & $15,7 \%$ & Composición & $45,0 \%$ \\
\hline & & Derivación & $1,70 \%$ \\
\hline
\end{tabular}

También se notan diferencias entre ambos tipos de corpus. Lo estable es la ventaja de la acronimia, que la ubica como el procedimiento preferido. Pero en el corpus diacrónico la composición presenta un peso importante, ya que abarca un $45 \%$ de las preferencias, cifra que baja a $15,7 \%$ en el corpus sincrónico, ya que los acrónimos suben hasta el 84,3\%. La derivación tiene una baja presencia, ya que involucra solo un caso del uso del prefijo mega, caso que se detectó después de constituido el corpus. 
Grado de unión gráfica

Tabla 13: unión gráfica

\begin{tabular}{|l|l|l|r|}
\hline \multicolumn{2}{|c|}{ C. sincrónico } & \multicolumn{2}{c|}{ C. diacrónico } \\
\hline Yuxtaposición & $62,7 \%$ & Yuxtaposición & $83,3 \%$ \\
\hline Disyunción & $37,3 \%$ & Disyunción & $11,7 \%$ \\
\hline & & Contraposición & $5,0 \%$ \\
\hline
\end{tabular}

Lo más llamativo en la comparación es que la contraposición ha desaparecido en el año 2008, frente al 5\% que muestra en el corpus diacrónico. Esto coincide con el poco uso que tiene, en general, el guión en las palabras compuestas.

Además, se percibe un aumento de la disyunción, la que en el corpus diacrónico tiene una baja aparición (11,7\% frente a un 37,3\%).

\section{A MODO DE CONCLUSIÓN}

En términos generales, podemos señalar que nuestro planteamiento inicial acerca de la existencia de palabras con gran poder condensador en textos escritos redactados para un receptor masivo fue confirmado, ya que en este estudio hemos reunido un corpus significativo ( 175 casos en total) en las muestras sincrónica (102 ejemplos en 2004-2007) y diacrónica (73 ejemplos entre 1975 y 2001). Esta indagación nos permitió comprobar que esta tendencia sintetizadora se ha ido ampliando con el paso del tiempo y que está en proceso de estandarización. Los corpora sincrónico y diacrónico fueron objeto de análisis desde varias perspectivas: sintáctica, morfológica, lexicogenésica, semántica y contextual; con el propósito de brindar una descripción lo más exhaustiva posible del uso innovador de los procesos de composición y acronimia en textos escritos públicos, en la actualidad, para generar así palabras que no existían en el fondo léxico de la lengua española, pero que son fácilmente comprensibles gracias al contexto en los que se emplean. A pesar de la indiscutible preeminencia de la derivación en los procesos de creación léxica del español, la composición y, sobre todo, la acronimia, tienen una vitalidad nada despreciable dentro del discurso publicitario escrito, debido a su poder de síntesis y eficacia comunicativa. 
Para finalizar, podemos describir el corpus de voces recopilado del siguiente modo:

- se trata de palabras con gran poder sintetizador, que contienen en sí una información susceptible de expresarse con un mayor número de unidades léxicas;

- el enunciado resultante, en cuanto a su superficie, carece de preposiciones y de varias clases de palabras (principalmente conectores) que deben ser reconstituidas por el receptor, así como las relaciones y funciones sintácticas y semánticas correspondientes;

- el contexto situacional es necesario en un nivel entre mediano y alto para la comprensión cabal del enunciado;

- el uso de estas construcciones implica una notable economía lingüística;

- la dependencia del contexto genera un rasgo opuesto a la economía lingüística, como lo es un mayor procesamiento cognitivo por parte del receptor del mensaje, quien debe interpretar adecuadamente una de estas palabras sintetizadoras;

- el procedimiento para constituir este tipo de palabras es, en primer lugar, la acronimia; y, en segundo lugar, la composición;

- estas palabras condensadoras figuran principalmente en textos publicitarios y propagandísticos escritos;

- primero, las palabras compuestas y los acrónimos analizados surgieron en un ámbito más informal y agresivo, desde el punto de vista comunicativo, como lo es la publicidad comercial, la que acude a recursos más variados con el objeto de promocionar un producto. Recién en el corpus de 20042007 se aprecian casos en anuncios institucionales (propagandísticos), lo cual constituye una prueba del avance del fenómeno hacia niveles de habla más formales;

- su escritura no está fijada y muestra formas alternativas que presentan variaciones en el uso de espacio intermedio, colores y mayúsculas, sin que el uso del guión sea un rasgo significativo;

- el uso de los elementos gráficos señalados arriba es cambiante y no significativo, ya que coexisten con el mismo significado palabras escritas con mayúscula inicial, con mayúscula en el segundo formante además del primero o con versalita;

- el nivel de complejidad de la información subyacente ha aumentado. Este aspecto se hizo claro al momento de explicitar esta información, ya que en el corpus diacrónico era más breve y directa (con relaciones de 
simple atribución), mientras que en el corpus sincrónico se vio un nivel más alto de motivación morfológica (Ullmann 1968) junto a relaciones internas complejas, en las que hay una predicación elaborada;

- la clase de palabras que participa en mayor medida es la sustantiva; y dentro de los sustantivos, la que se reveló más productiva fue fono, acortamiento de teléfono;

- la perspectiva diacrónica muestra un aumento sostenido desde 1975 hasta 2008, lo que refleja una preferencia notable de los autores de textos publicitarios por estas palabras sintetizadoras.

\section{REFERENCIAS BIBLIOGRÁFICAS}

Almela, Ramón. 1999. Procedimientos de formación de palabras en español. Barcelona: Ariel.

Alvar, Manuel. 1996. La formación de palabras en español. Madrid: Arco Libros.

Casado, Manuel. 1999. Otros procedimientos morfológicos: acortamientos, formación de siglas y acrónimos. En Ignacio Bosque y Violeta Demonte (dirs.), Gramática descriptiva de la lengua española, Vol. 3. Madrid: Espasa Calpe, pp. 5075 - 5096.

Ferraz, Antonio. 2000. El lenguaje de la publicidad. Madrid: Arco Libros.

Fernández, Ángel, Salvador Hervás y Valerio Báez.1984. Introducción a la semántica. Madrid: Cátedra.

Lang, Mervyn. 1997. Formación de palabras en español. Madrid: Cátedra.

Martinet, ANDré. 1984. Estudios de sintaxis funcional. Madrid: Gredos.

Real Academia Española. 1999. Ortografía de la Lengua Española. Madrid: Espasa-Calpe S.A.

2001. DRAE. Diccionario de la lengua española (Vigésima segunda edición). En línea: http://www.rae.es [Consulta 01/03/2010].

Ullmann, Stephen. 1968. Semántica. Madrid: Aguilar.

Varela, Soledad. 2005. Morfología léxica: la formación de palabras. Madrid: Gredos. 
CORPUS SINCRÓNICO (resumido)

\begin{tabular}{|l|l|l|l|}
\hline \multicolumn{3}{|l}{ Muestra } \\
\hline 1 & AutoPlaza & 30 & Fono Salo \\
\hline 2 & BancoEstado & 31 & Fono sed \\
\hline 3 & BankBoston & 32 & FONO SERVICIO \\
\hline 4 & Banmedica & 33 & Fono Servicio \\
\hline 5 & (Concurso) Chilelnventa & 34 & FONO SOUR \\
\hline 6 & (GMO) Econópticas & 35 & FONO TAG \\
\hline 7 & ENTRELATAS & 36 & Fono taxi Aries \\
\hline 8 & Feriaticket & 37 & Fono taxi El Molino \\
\hline 9 & FONO ACOGIDA & 38 & Fono Taxi Emitell \\
\hline 10 & Fono Ayuda & 39 & Fono Taxi Lo Franco \\
\hline 11 & FONO AYUDA & 40 & Fono Taxi Metropolitano \\
\hline 12 & FONO CAMINO & 41 & Fono Taxi Plaza Valdivieso \\
\hline 13 & FONO CLIENTE & 42 & Fono Taxi Renca \\
\hline 14 & Fonocliente & 43 & Fono Taxi San Joaquín \\
\hline 15 & Fono Compras & 44 & Fono Taxis San José \\
\hline 16 & Fono Consulta & 45 & FONO VENTAS \\
\hline 17 & Fono consulta & 46 & Fono Ventas \\
\hline 18 & Fono Consultas & 47 & Fonos Ventas \\
\hline 19 & FONO DENUNCIA & 48 & FONO VENTAS \\
\hline 20 & FONO DROGAS & 49 & Fonocenter \\
\hline 21 & FONO EMERGENCIA & 50 & FONOCENTRAL \\
\hline 22 & Fono Gratis 24 hrs & 51 & FONOCLIENTE \\
\hline 23 & FONO NIÑOS & 52 & FonoCompras \\
\hline 24 & FONO OFRENDA & 53 & FONOCOMPRAS \\
\hline 25 & FONO PAGO & 54 & Fonocompras \\
\hline 26 & FONO PIZZA & 55 & Fonoconsulta \\
\hline 27 & FONO RECLAMO & 56 & FonoIANSA \\
\hline 28 & Fono reparto & 57 & Fonomujer \\
\hline 29 & FONO RESERVA & 58 & FonoPresto \\
\hline
\end{tabular}




\begin{tabular}{|l|l|l|l|}
\hline 59 & FONORATAS & 91 & Publiguías \\
\hline 60 & Fonoreserva & 92 & Pucmático \\
\hline 61 & Fonoreserva & 93 & (El) Quitapenas \\
\hline 62 & FonoServicio & 94 & RedCompra \\
\hline 63 & FonoServicio & 95 & Servipag \\
\hline 64 & FonoServicio & 96 & Sirvefácil \\
\hline 65 & FonoServicio & 97 & SOLODIVORCIOS \\
\hline 66 & FONOSIDA & 98 & SuperHipoteca \\
\hline 67 & Fonotaxi Lo Castillo & 99 & Telemergencia \\
\hline 68 & Fonotaxi El Molino & 100 & TELEPEAJE \\
\hline 69 & Fonotaxi Montserrat & 101 & Telepizza \\
\hline 70 & Fonotaxi Unimarc & 102 & (Equipos de) videovigilancia \\
\hline 71 & Fonotaxis & & \\
\hline 72 & FONOTIMBRE & & \\
\hline 73 & Fonoviajes & & \\
\hline 74 & FONOVIAJES & & \\
\hline 75 & Fotoamérica & & \\
\hline 76 & FOTOAVISOS & & \\
\hline 77 & FOTOBORDE & & \\
\hline 78 & FOTORETOQUE & & \\
\hline 79 & FOTOSTICKERS & & \\
\hline 80 & Infocentros & & \\
\hline 81 & INSTANFONO & & \\
\hline 82 & INSTANPARIS & & \\
\hline 83 & Masvida & & \\
\hline 84 & Metrored & & \\
\hline 85 & Multioportunidad & & \\
\hline 86 & (Club) Niño Ahorro & & \\
\hline 87 & Previred & & \\
\hline 88 & Prontofertas & & \\
\hline 89 & Provifono & & \\
\hline 90 & Provimático & & \\
\hline
\end{tabular}


CORPUS DIACRÓNICO (resumido)

\begin{tabular}{|l|l|l|l|}
\hline \multicolumn{3}{|c|}{ Muestra } \\
\hline 1 & afeitabic & 31 & FRIOMARKET \\
\hline 2 & AHORROFERTAS & 32 & FULL PIZZA \\
\hline 3 & AUTOCAR & 33 & INFODROGA \\
\hline 4 & AUTONOR & 34 & LanChile \\
\hline 5 & autoparts & 35 & LANPASS \\
\hline 6 & BancoEstado & 36 & LAPAPA \\
\hline 7 & BanRenta & 37 & Megavisión \\
\hline 8 & cablexpress & 38 & METROGAS \\
\hline 9 & CASAROYAL & 39 & MOVICENTER \\
\hline 10 & CENTROofertas & 40 & Multiofertas \\
\hline 11 & CHEQUEcompra & 41 & MUNDO-MÁGICO \\
\hline 12 & CHILEMILLONARIO & 42 & (OFERTAS) SUPEREXTRAS \\
\hline 13 & CHILESAT & 43 & PORMAYOR \\
\hline 14 & Chilevisión & 44 & Pre-Unic \\
\hline 15 & Cinecanal & 45 & RADIOZERO \\
\hline 16 & construmart & 46 & Redbanc \\
\hline 17 & CONVIDA & 47 & RENTAMáS \\
\hline 18 & CORPBANCA & 48 & SERVI-FRENOS \\
\hline 19 & CREDISUR & 49 & SERVIRENTAS \\
\hline 20 & DATO-AHORRO & 50 & SuperTeléfono \\
\hline 21 & DIS-CARD & 51 & TANGOMANIA \\
\hline 22 & Electromat & 52 & Tecnicentro (Sierra) \\
\hline 23 & ELECTRO-SALUD & 53 & Tecnicolor \\
\hline 24 & Entelchile & 54 & TecnoMAR \\
\hline 25 & Entelticket & 55 & Telecable \\
\hline 26 & EXTRACREDITO & 56 & TELENORTE \\
\hline 27 & FACILPLAN & 57 & (centro de atención) \\
\hline 28 & FANTASILANDIA & 58 & TOdaHORA \\
\hline 29 & FENSA-MADEMSA & 59 & TODO \\
\hline 30 & Fono-crédito & 60 & TUR BUS \\
\hline
\end{tabular}

